Seventh Meeting, 10th June 1904.

Mr Charles Tweedie, President, in the Chair.

\title{
The Turning-Values of a Cubic Function and the nature of the roots of a Cubic Equation.
}

By P. Pinkerton, M.A.

The first part of this paper depends on the theorem that if $a, b, c$ are three positive quantities such that

$$
a+b+c=\mathbf{a} \text { constant, }
$$

then $a b c$ is a maximum when $a=b=c$; with the corollary that if $a, b, c$ are three negative quantities such that

$$
a+b+c=\text { a constant, }
$$

then $a b c$ is a minimum when $a=b=c$.

1. Consider the graph of

where $a$ is positive.

$$
y=x(x-a)^{2},
$$

The graph meets $\mathrm{OX}$ at the points $(0,0)(a, 0)$. The graph has a minimum point at $(a, 0)$, for on shifting the origin to $(a, 0)$ the equation becomes

$$
y=(\xi+a) \xi^{2}
$$

and a frst approximation at the new origin is

$$
y=a \xi^{2} \text {, }
$$

so that the graph close to that point is of the form of a festoon. There is also a maximum point in the interval between $x=0$ and $x=a$. To determine the point we observe that $x(x-a)^{2}$ is a maximum when $2 x(a-x)(a-x)$ is a maximum. Now each of these factors is positive in the interval considered and their sum is constant $(=2 a) ; \therefore$ a maximum value occurs when

$$
2 x=a-x \text { i.e., when } x=\frac{a}{3} .
$$

The maximum value is therefore $\frac{a}{3}\left(\frac{a}{3}-a\right)^{2}=\frac{4 a^{3}}{27}$. 
2. Again considering the graph of

$$
y=x(x+a)^{2}
$$

where $a$ is positive, we observe that if the origin is shifted to the point $(-a, 0)$ a first approximation at the new origin is

$$
y=-a \xi^{2}
$$

which represents an inverted festoon. There is therefore a maximum point at $(-a, 0)$ and a minimum point in the interval $x=0$ to $x=-a$. To find this point we observe that $x(x+a)^{2}$ has its minimum value when $2 x(-a-x)(-a-x)$. is a minimum. Each of these factors is negative in the interval considered and their sum is constant $(=-2 a)$;

$$
\begin{aligned}
& \therefore \quad 2 x=-a-x \text { for the minimum point; } \\
& \therefore \quad x=-\frac{a}{3} ;
\end{aligned}
$$

and the minimum value is $-\frac{4 a^{3}}{27}$.

3. In general let

$$
y=x^{3}+p x^{2}+q x+r=(x+a)(x+b)^{2}+c .
$$

Equating coefficients, we have

$$
\begin{aligned}
& p=a+2 b, \\
& q=2 a b+b^{2}, \\
& r=a b^{2}+c .
\end{aligned}
$$

Eliminating $a$ from (1) and (2),

$$
3 b^{2}-2 b p+q=0
$$

$\therefore$ real values of $a, b, c$ can be found if

$$
p^{2} \equiv 3 q \text {. }
$$

If $p^{2}=3 q$, it is clear that we can write

$$
y=x^{3}+p x^{2}+q x+r=\left(x+\frac{p}{3}\right)^{3}+\left(r-\frac{p^{3}}{27}\right),
$$

and by changing the origin to $\left(-\frac{p}{3}, r-\frac{p^{3}}{27}\right)$ the equation takes the form $\eta=\xi^{3}$ which has a point of inflexion at the new origin and no turning-points. 
If $p^{2}>3 q, y=(x+a)(x+b)^{2}+c$ where $a, b, c$ are real. Shift the origin to $(-a, c)$ and the equation becomes

$$
\eta=\xi(\xi-\overline{a-b})^{2} .
$$

Hence, if $a-b$ is positive, there is a minimum turning-point at

$$
\xi=a-b, \eta=0 \text { i.e, at } x=-b, y=c .
$$

Also there is a maximum turning-point at

$$
\begin{gathered}
\xi=\frac{1}{3}(a-b), \eta=\frac{4}{37}(a-b)^{3} \\
i . e ., \text { at } x=-a+\frac{1}{3}(a-b), y=c+\frac{4}{27}(a-b)^{3} .
\end{gathered}
$$

If $(a-b)$ is negative, there is a maximum turning-point at

$$
\xi=a-b, \eta=0 \text { i.e., at } x=-b, y=c
$$

and a minimum turning-point at

$$
\begin{gathered}
\xi=\frac{1}{3}(a-b), \eta=\frac{4}{27}(a-b)^{3} \\
\text { i.e., at } x=-a+\frac{1}{3}(a-b), y=c+\frac{4}{27}(a-b)^{3} .
\end{gathered}
$$

The graph whose equation is

$$
y=\mathbf{A} x^{3}+p x^{2}+q x+r
$$

can clearly be reduced to the above case.

4. The nature of the roots of a cubic equation can be deduced from our knowledge of the above graphs.

Suppose the equation first brought to the form

$$
x^{3}+q x+r=0 \text {. }
$$

Let

$$
y=x^{3}+q x+r=(x+a)(x+b)^{2}+c .
$$

Here

$$
\begin{aligned}
a+2 b & =0, \\
2 a b+b^{2} & =q, \\
a b^{2}+c & =r .
\end{aligned}
$$

If two roots are equal then clearly $c=0$;

$$
\begin{aligned}
& \therefore \text { by }\left(1^{\prime}\right) \text { and }\left(2^{\prime}\right) \quad-3 b^{2}=q \text {, } \\
& \text { and by }\left(1^{\prime}\right) \text { and }\left(3^{\prime}\right) \quad-2 b^{3}=r \text {; } \\
& \therefore \quad 4 q^{3}+27 r^{2}=0 .
\end{aligned}
$$

If the three roots are unequal $c \neq 0$.

In this case it is clear that if $a_{1}, b_{1}, c_{1}$ and $a_{2}, b_{2}, c_{2}$ are the two sets of solutions of $\left(1^{\prime}\right),\left(2^{\prime}\right),\left(3^{\prime}\right)$, then

$$
\begin{aligned}
& a_{1}+a_{2}=0, b_{1}+b_{2}=0 ; \\
& \therefore \quad\left(a_{1}-b_{1}\right)=-\left(a_{2}-b_{2}\right) .
\end{aligned}
$$


Suppose $a_{1}-b_{1}$ to be positive and write

$$
y=\left(x+a_{1}\right)\left(x+b_{1}\right)^{2}+c_{1} .
$$

It follows from the above that the minimum turning value is given by $y=c_{1}$.

Next, writing $y=\left(x+a_{2}\right)\left(x+b_{2}\right)^{2}+c_{2}$, we observe that the maximum turning value is given by

$$
y=c_{2}
$$

$\therefore \quad$ OX will cut the graph of

$$
y=x^{3}+q x+r
$$

in three different real points if $\frac{c_{1}}{c_{2}}$ is negative,

and in one real point and two imaginary points if $\frac{c_{1}}{c_{2}}$ is positive.

There are therefore three different real solutions of the equation, or one real and two imaginary,

$$
\begin{aligned}
& \text { according as } \frac{c_{1}}{c_{2}} \quad \text { is negative or positive, } \\
& \therefore \quad \text { " } \quad \frac{c_{1}{ }^{2}}{c_{1} c_{2}} \quad " \quad " \quad " \quad " \\
& \therefore \quad " \quad c_{1} c_{2} \quad " \quad " \quad " \quad " \\
& \therefore \quad " \quad,\left(r-a_{1} b^{2}\right)\left(r-a_{2} b^{2}\right), \quad, \quad, \quad, \\
& \therefore \quad " \quad " \quad r^{2}+a_{1} a_{2} b^{4} \quad, \quad " \quad, \quad "\left(\because a_{1}+a_{2}=0\right) \\
& \therefore \quad " \quad, \quad r^{2}-a^{2} b^{4} \quad, \quad, \quad " \quad " \\
& \therefore \quad " \quad \text { " } r^{2}-4 b^{8} \quad \text { " } \quad \text { " } \quad \text { " by }\left(1^{\prime}\right) \\
& \therefore \quad " \quad \text { " } \quad r^{2}+\frac{4}{27} q^{3} \quad " \quad, \quad " \quad \text { by }\left(1^{\prime}\right) \text { and }\left(2^{\prime}\right) \\
& \therefore \text { according as } 4 q^{3}+27 r^{2} \text { is negative or positive. }
\end{aligned}
$$

Note on the Problem: To draw through a given point a transversal to $(a)$ a given triangle $(b)$ a given quadrilateral so that the intercepted segments may have (a) a given ratio (b) a given cross ratio.

$$
\text { By P. Pinkerton, M.A. }
$$

